\title{
EVALUATING EXPECTED PERFORMANCE AND GRACEFUL DEGRADATION IN DISTRIBUTED SPACECRAFT MISSIONS
}

\author{
Afreen Siddiqi ${ }^{1}$, Jacqueline Le Moigne ${ }^{2}$ \\ ${ }^{1}$ Massachusetts Institute of Technology, Cambridge, MA, USA \\ ${ }^{2}$ NASA Goddard Space Flight Center, Greenbelt, MD, USA
}

\begin{abstract}
Distributed Spacecraft Missions (DSMs) have largely garnered interest due to lower cost and new technical capabilities. However, another important attribute in DSMs is the potential of graceful degradation where a failure of one or more spacecraft (or instruments) in the DSM can reduce total performance, but the remaining functional spacecraft can maintain a limited science return. The potential for retaining some mission value in the event of partial failures leads to enhanced lifetime value. Here, an approach is developed to analyze graceful degradation and performance of DSM used for Earth observation. The system performance is defined through metrics of coverage and average revisit time of points of interest. Graceful degradation is quantified through total change and rate of change in performance metrics with increasing sub-system failures over the lifetime of a mission. An Expected Performance measure is formulated for conducting comparison of DSM architectures for early stage conceptual mission design and trade studies.
\end{abstract}

Index Terms - Distributed Space Missions, Expected Performance, Graceful Degradation, Science Return, Space Systems Architecture.

\section{INTRODUCTION}

Distributed Spacecraft Missions (DSMs), involving multiple spacecraft to achieve one or more common goals, offer unique advantages for increasing and enhancing science return from earth observation missions. DSMs, which we will also refer to as 'constellations' here, not only can simultaneously increase observation sampling in spatial, spectral, temporal and angular dimensions that enable new scientific investigation, but also mitigate mission risk and facilitate data continuity and enhance total science return over the mission lifetime [1]. The growing focus on DSMs stems from a combination of budget cuts, maturing technologies and new science requirements. As DSMs can consist of smaller and lighter spacecraft (as compared to traditional monolith earth observation spacecraft), which can be deployed through smaller launch vehicles or through secondary launches, the total mission costs can be significantly lower. Additionally, instrument and component miniaturization are enabling new research in Earth Science.

While DSMs have largely garnered interest due to lower cost and promise of new technical capabilities, there are other attributes that can add to their importance such as enhanced lifetime value delivery [2], reconfigurability [3], and flexibility [4]. Research in defining, quantifying, and valuing such properties in complex systems in general (and to some extent distributed space systems in particular) has been conducted. However, there has been limited work (and no widely used approaches) that focus specifically on DSMs.

In this work we develop a framework to quantify and analyze expected performance in the event of partial losses within the distributed system of DSMs, and discuss how this attribute of graceful degradation can be used for comparative assessment and architecture analysis in trade studies for pre-phase A earth observation mission design.

\section{LITERATURE REVIEW}

The use of constellations for services such as navigation, communication and earth imaging have steadily and consistently increased in the past decades. For earth and space science missions, there have been a number of concepts proposed and studied for distributed spacecraft missions [5], and some have now been deployed [6] while others are advancing in development.

In addition to advantages in expanded coverage and spatial and temporal resolution that a distributed architecture can provide, there are a number of new system level characteristics that can add to the overall value obtained from the mission over its operational lifetime. The Defense Advanced Research Project Agency (DARPA) commissioned a series of studies and research projects on investigating the value of 'Fractionated Spacecraft Missions' wherein the functional capabilities of a conventional monolithic spacecraft are distributed across multiple modules that are not structurally connected and that interact through wireless links [7]. Some key sources of added-value and appeal for such architectures that were identified included flexibility for staged deployment (that could allow for decoupling payload development timelines and also help in distributing and thus reducing cost), 
simplifying payload to spacecraft integration, mitigating impact of launch failures, degrading gracefully due to onorbit failures, having the possibility to 'replace' a failed module and thus 'repairing' and recovering, and scaling (or adding additional modules) to expand capabilities as needed [7]. A detailed analysis, however, showed that there are a number of factors that can add to costs such as the need for multiple launches, increased operational complexity, intraspacecraft inefficiencies and others that can collectively make the value proposition uncertain.

In separate studies, researchers analyzed how replacement of failed modules (that is possible with a fractionated architecture) impacts the cost and utility comparison of monolith and fractionated (or networked) architectures for space missions [8]. The analysis used Markov models for failure and replacement analysis and Monte-Carlo simulations to study how the architectures perform on cost, utility, and utility per unit cost basis [8].

\section{EXPECTED PERFORMANCE OF DSM}

For earth observation missions, we define the value delivering function as the acquisition and transmission of data as needed for meeting the science goals and mission objectives. For early stage conceptual mission design studies, some relevant key performance measures (that relate to the value delivering function) are maximum and average revisit time for points of interest (POI), and spatial coverage and resolution. The lifecycle cost and risk are measures that are used to evaluate tradeoffs between different options for architectures with different performance.

\subsection{Performance Measures}

In this analysis, we use coverage, average and maximum revisit time of POI, and per unit cost of science data to characterize performance of an architecture. The cost per data (CPD) metric is defined as the ratio of total data successfully transmitted for scientific use to the lifecycle cost of the mission. This metric is similar to the cost per function (CPF) measure used in earlier studies of distributed spacecraft [5], and utility per unit cost for fractionated spacecraft [8].

\subsection{Graceful degradation}

Graceful degradation, where the functionality or utility of the system gradually declines due to partial failures in constituent elements or sub-systems, is an important attribute of distributed architectures. Systems that consist of multiple elements can continue to have limited functionality even when some elements become inoperative. In the case of DSMs, a failure of one or more spacecraft (or instruments) in the constellation can lead to reduced performance, but it may be possible to maintain a limited (though degraded) science return from the mission.

Graceful degradation can be modeled using Markov theory, wherein a system is defined to exist within a set of finite states, and it transitions between states as a result of stochastic events (such as failures).

In this analysis, we use Markov modeling to quantify change in performance measures as a result of on-orbit failures of elements within a DSM. We assume that the architecture of a DSM consisting of $s$ spacecraft is defined within a specified set of orbital parameters, and it carries payloads of given specifications, and has a design lifetime of $\mathrm{T}_{\text {life }}$. We define a set of states of the system (the DSM) such that each state represents a condition wherein some failures have occurred in one or more of the $s$ spacecraft comprising the DSM. The initial state is one where the system is fully functioning (with all of its constituent elements performing as designed), and the final state is where all of the $s$ spacecraft have failed. The nature and source of failure in spacecraft can vary (ranging from failures in critical subsystems including power, attitude control, data handling and processing, or the instrument payload).

For purposes of early stage analysis and architecture trade studies, it is sufficient to model failure at the spacecraft level where the 'failure' refers to a state in which the spacecraft is unable to provide data as designed and desired for the mission. The probability of the system to be in a state $i$ is denoted as $\pi_{\mathrm{i}}$, and the transition rate of changing from a state $i$ to a state $j$ is denoted as $\lambda_{\mathrm{ijj}}$. For a continuous time model, the following equations hold [9]:

$$
\begin{aligned}
& \frac{d}{d t} \Pi(t)=A \Pi(t) \\
& \Pi(t)=e^{A t} \Pi_{0}
\end{aligned}
$$

where $\Pi_{0}$ is the initial state vector, and $\Pi(t)$ is the vector consisting of elements $\pi_{\mathrm{i}}$ at time $\mathrm{t}$, and $\mathrm{A}$ is the transition matrix.

The performance degradation of the DSM can be assessed using the performance metrics of the system. For a performance metric $\rho_{i}$, the expected performance is defined as:

$$
\begin{aligned}
& E[\rho]=\rho^{T} \Pi(t) \\
& \text { where } \rho^{T}=\left[\begin{array}{lllll}
\rho_{1} & \ldots & \rho_{1} & \ldots & \rho_{n}
\end{array}\right]
\end{aligned}
$$

for $n$ states of the system and $\rho_{i}$ is the performance of the system when it is in state $i$. The expected performance and change in performance within a given time window allow for quantitatively distinguishing between architectures (as shown in Figure 1 for a notional case). The figure illustrates that the change in performance for the first 
architecture is $30 \%$ while for the second architecture is $20 \%$ in a two year time duration.

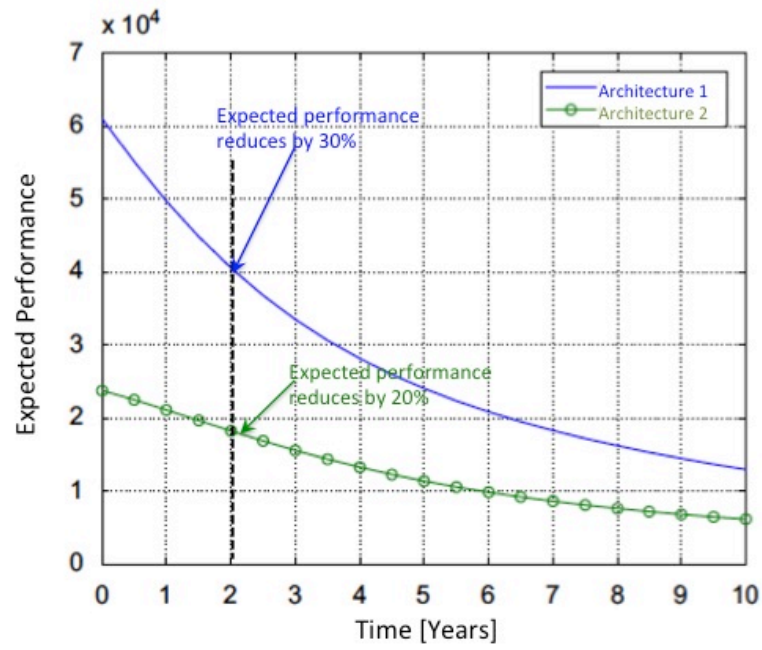

Figure 1: Expected performance over mission lifetime for two illustrative architecture cases.

The rate of change in performance between time $t_{1}$ and $t_{2}$ can be computed as $\delta_{12}$ for a given architecture:

$$
\delta_{12}=\frac{E[\rho]_{t_{2}}-E[\rho]_{t_{1}}}{t_{2}-t_{1}}
$$

\section{COMPARATIVE ANALYSIS AND TRADE STUDIES FOR DSM}

The traditional approach in Markov analysis is to study system behavior over time, and typical attributes of interest are how much time a system may spend in a given state and how quickly a system transitions to a particular state. In the case of architecture trade space analysis for DSMs, in addition to these properties, it is also useful to compare how the system performs within different states (regardless of when those states are achieved). This allows one to assess what the system performance becomes when one or more spacecraft fail (irrespective of when the failures may occur). Figure 2 conceptually illustrates how the three performance metrics described in section 3.1 can be used for comparative analysis and trade studies.
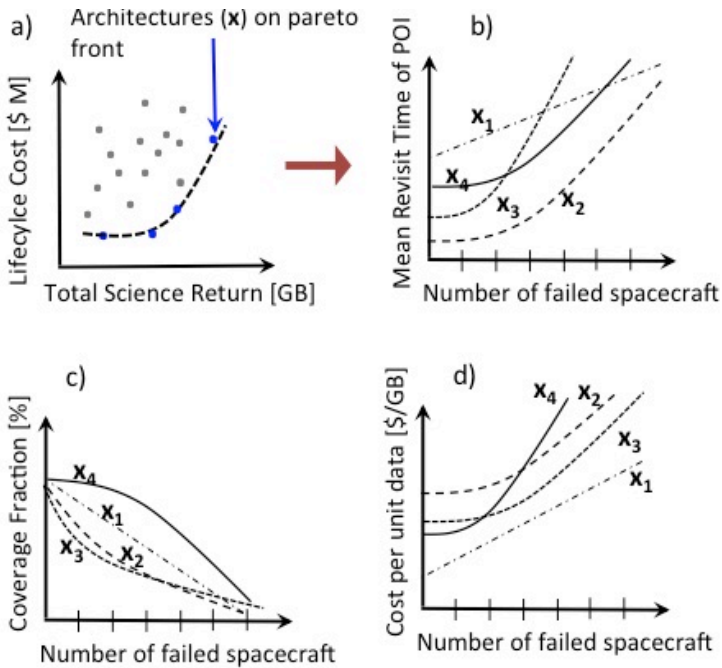

Figure 2: Performance metrics of revisit time, coverage fraction and cost per unit data are evaluated for partially failed elements in a set of four DSM architectures (denoted as $x_{i}$ ).

This approach allows for comparing total, rate of change in performance, and thresholds (number of failures after which the mission no longer provides any science return or value for operators and users). The performance measures are computed with modeling tools with necessary capabilities to analyze earth observation missions given a set of specifications and design parameters.

\section{APPLICATION: TRADE-SPACE ANALYSIS TOOL FOR CONSTELLATIONS (TAT-C)}

The Trade space Analysis Tool for Constellations (TAT-C) has been developed to provide a framework for conducting pre-Phase A mission analysis of DSMs. It allows for modeling multiple spacecraft sharing a mission objective, and helps explore trade-space of variables for pre-defined science, cost and risk goals and metrics [1].

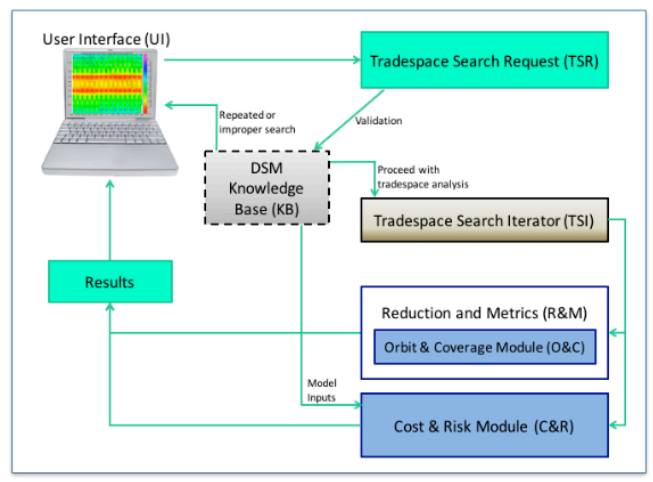

Figure 3: Trade-space Analysis Tool for Constellations (TAT-C) [ref] 
TAT-C models orbits and coverage for spacecraft using semi-analytic orbit propagation using osculating Keplerian elements. It computes performance of architectures over mission lifetime, and outputs minimum, maximum and average information across all POIs, information per POI and information as a time series. These outputs can be combined to provide mission level measures such as percentage POI covered, revisit times etc. These outputs will be used in conjunction with Markov analysis to compute and compare DSM architectures for a given set of scientific goals.

In continuing work, we will use TAT-C to demonstrate computation of expected performance of DSMs, and will also investigate how different architectures compare on the measures of graceful degradation and lifetime mission value for a given class of earth observation missions.

\section{SUMMARY}

This paper introduces a framework for studying graceful degradation in distributed space missions using Markov analysis. A set of performance measures are proposed that are relevant for earth observation missions, and the expected performance metric is proposed as a basis for quantitative comparison and trades between architectures during early stages of mission design analysis and exploration.

The application of this approach is discussed with a new framework, the Trade-space Analysis Tool for Constellations (TAT-C), currently being developed at NASA Goddard Space Flight Center, that will enable the design of future Distributed Spacecraft Missions (DSM). The key attributes currently simulated are cost, risk and coverage metrics. The addition of expected performance measures as a function of partial losses (which is an advantage of distributed architectures) will expand the analytical capabilities of TAT-C and will provide important assessment of attributes that are unique to DSMs.

\section{ACKNOWLEDGEMENT}

The authors wish to thank the support of the Earth Science Technology Office (ESTO) for funding by the Advanced Information Systems Technology (AIST) program.

\section{REFERENCES}

[1] J. LeMoigne, P. Dabney, O. DeWeck, V. Foreman, P. Grogan, M. Holland, S. Hughes, S. Nag, and A. Siddiqi, "Trade-space Analysis Tool for Designing Earth Science Distributed Missions TAT-C: Tradespace Analysis Tool for Constellations," 2018.

O. De Weck, R. De Neufville, and M. Chaize,
"Staged Deployment of Communications Satellite Constellations in Low Earth Orbit," J. Aerosp. Comput. Information, Commun., vol. 1, no. March, pp. 119-136, 2004.

[3] A. Siddiqi, "System Reconfigurability," in Encyclopedia of Aerospace Engineering, R. Blockley and W. Shyy, Eds. John Wiley and Sons, 2010.

[4] A. M. Ross, D. H. Rhodes, and D. E. Hastings, "Defining Changeability: Reconciling Flexibility, Adaptability, Scalability, Modifiability, and Robustness for Maintaining System Lifecycle Value," Syst. Eng., vol. 11, no. 3, pp. 246-262, 2008.

[5] G. B. Shaw, "Development of the Quantitative Generalized Information Network Analysis (GINA) Methodology for Satellite Systems," J. Spacecr. Rockets, vol. 38, no. 2, pp. 257-269, 2001.

[6] R. Rose, C. Ruf, J. Scherrer, and J. Wells, "The CYGNSS Flight Segment ; Mainstream Science on a Micro-Budget," in Aerospace Conference, 2015 IEEE, 2015.

[7] D. B. Maciuca, J. K. Chow, A. Siddiqi, O. L. De Weck, S. Alban, L. D. Dewell, A. S. Howell, J. M. Lieb, B. P. Mottinger, J. Pandya, M. J. Simon, P. P. Yang, A. L. Zimdars, S. I. Saeed, J. Ramirez, A. Saenz-Otero, D. W. Miller, and G. S. Hubbard, “A Modular, High-Fidelity Tool to Model the Utility of Fractionated Space Systems," in Encyclopedia of Aerospace Engineering, R. Blockley and W. Shyy, Eds. John Wiley and Sons, 2010.

[8] G. F. Dubos and J. H. Saleh, "Comparative cost and utility analysis of monolith and fractionated spacecraft using failure and replacement Markov models," Acta Astronaut., vol. 68, no. 1-2, pp. 172184, 2011.

[9] W. Feller, An Introduction to Probablity Theory and Its Applications. Wiley, 1968. 